\title{
Efficacy of pemetrexed-based regimen in relapsed advanced thymic epithelial tumors and its association with thymidylate synthetase level
}

This article was published in the following Dove Press journal:

OncoTargets and Therapy

22 July 2016

Number of times this article has been viewed

\author{
Xinyu Qian ${ }^{1, *}$ \\ Zhengbo Song $2,3, *$ \\ 'Department of Chemotherapy, \\ Hangzhou First People's Hospital, \\ Hangzhou Cancer Hospital, Hangzhou, \\ ${ }^{2}$ Key Laboratory Diagnosis and \\ Treatment Technology on Thoracic \\ Oncology, Zhejiang Province, \\ ${ }^{3}$ Department of Chemotherapy, \\ Zhejiang Cancer Hospital, Hangzhou, \\ People's Republic of China \\ *These authors contributed equally \\ to this work
}

Correspondence: Zhengbo Song Department of Chemotherapy,

Zhejiang Cancer Hospital, 38

Guangji Road 310022, Hangzhou,

People's Republic of China

Tel +8657188122182

Fax +86571 8812 2188

Email songzhengbo83@।63.com
Purpose: Due to the rarity of thymic epithelial tumors (TET), no standard chemotherapy regimen has been identified in the relapsed setting. The aim of this study was to investigate the activity of a pemetrexed-based regimen in advanced TET as palliative treatment after failure of previous chemotherapy, and to detect its association with thymidylate synthetase (TS) level.

Methods: Patients with pathologically confirmed TET and treated with pemetrexed-based regimen were evaluated from 2006 to 2014 in Zhejiang Cancer Hospital. TS mRNA level was detected by real-time polymerase chain reaction. The Kaplan-Meier method was used for survival analysis.

Results: A total of 22 TET patients were identified, of whom eight had thymoma and 14 had thymic carcinoma. In total, the objective response rate and disease control rate of the 22 patients were $22.7 \%$ and $68.2 \%$, respectively. Median progression-free survival and overall survival were 4.5 months and 34.9 months, respectively. A trend of lower TS mRNA levels existed in patients with disease control compared to those with progressive disease $\left(268.0 \pm 160.5 \times 10^{-4}\right.$ vs $\left.567.0 \pm 445.0 \times 10^{-4}, P=0.065\right)$.

Conclusion: Patients with advanced TET may benefit from pemetrexed-based regimen therapy. TS mRNA level is valuable for predicting the efficacy of pemetrexed in TET.

Keywords: thymic epithelial tumors, pemetrexed, salvage chemotherapy, efficacy, thymidylate synthetase

\section{Introduction}

Thymic epithelial tumors (TET) are relatively rare mediastinal neoplasms. ${ }^{1}$ Surgery is the standard treatment for patients at an early stage, and complete resection is the most important prognostic factor for survival. ${ }^{2}$ However, recurrence or metastasis is a big issue in clinical practice. ${ }^{3}$ For patients with distant metastasis, salvage chemotherapy plays an important role in the treatment of thymic malignancies. ${ }^{4,5}$ No definitive chemotherapeutic regimen has been established due to its rarity. The regimens of cisplatin, vincristine, doxorubicin, and etoposide; cisplatin and paclitaxel; and etoposide, ifosfamide, and cisplatin are recommended as first-line treatment with a progression-free survival (PFS) within 8 months. ${ }^{6-8}$ Most of the patients would progress after first-line chemotherapy. For these patients, standard regimens have not been identified.

Pemetrexed is a pyrrolopyrimidine antifolate, which inhibits thymidylate synthetase, glycinamide ribonucleotide formyltransferase, and dihydrofolate reductase. Pemetrexed is widely used for the treatment of nonsquamous non-small cell lung cancer (NSCLC) and malignant pleural mesothelioma. The results of randomized 
studies demonstrated that pemetrexed was less toxic and more effective in NSCLC treatment. ${ }^{9-11}$ However, the efficacy and toxicity data of pemetrexed as salvage therapy were limited in TET. In addition, thymidylate synthetase (TS) level was significantly associated with clinical efficacy of pemetrexed-based chemotherapy in NSCLC patients. ${ }^{12,13}$ However, the association between the TS level and efficacy of pemetrexed-based regimen in TET is unclear.

In this study, we conducted a retrospective study to evaluate the efficacy of pemetrexed-based regimen in advanced TET as salvage chemotherapy and further to detect the relationship between TS level and clinical efficacy of this regimen.

\section{Materials and methods Patient eligibility}

Data from 22 patients who received pemetrexed-based treatment as second- or further-line chemotherapy regimen with advanced TET were collected in Zhejiang Cancer Hospital from 2006 to 2014. All patients met the following criteria: 1) histological diagnosis of TET according to the histopathological criteria proposed by the World Health Organization 2004 version; 2) Masaoka criteria stage of IVa or IVb; 3) had progressed from prior chemotherapy regimens; 4) without any local treatment such as radiotherapy or interventional therapy during the time of pemetrexedbased therapy. The Ethics Committee of Zhejiang Cancer Hospital approved this study. All patients provided written informed consent.

\section{Efficacy evaluation}

Clinical efficacy was assessed every two cycles, or evaluated early when significant signs of progression appeared. The Response Evaluation Criteria in Solid Tumors (RECIST 1.1) was used to evaluate tumor responses.

\section{Measurement of TS mRNA}

Tumor cells were collected from formalin-fixated, paraffinembedded tissue samples. Total RNA was extracted using a commercial method (Amory, Xiamen, People's Republic of China), and cDNA was generated with oligo-dT and random primers. Real-time quantitative polymerase chain reaction analysis was performed in triplicate per sample. The relative amount of TS mRNA in a sample was determined by comparing the threshold cycle with a standard curve. The details of this process have been published in previous studies..$^{14,15}$

\section{Follow-up and statistical analysis}

The last follow-up time was March 31, 2015 and median follow-up period was 37.5 months (5.0-62). The follow-up rate was $100 \%$. Overall survival (OS) was defined as the time from the first day of diagnosis as advanced issues to death or last follow-up time. PFS encompassed the time from the first cycle of pemetrexed-based treatment to documented progression or death from any cause. The survival curves were calculated according to the method of Kaplan-Meier. Statistical analysis was performed using SPSS version 18.0 (SPSS Inc., Chicago, IL).

\section{Results}

\section{Patients' characteristics}

Twenty-two patients were included in this study. There were ten males and 12 females with median age of 48.5 years old. Eight patients had thymoma and 14 had thymic carcinoma. Twelve patients had a performance status (PS) of $0-1$ and ten had a PS of 2. Eighteen patients received single pemetrexed and four received pemetrexed/carboplatin therapy. The first-line regimens of the 22 patients comprised cyclophosphamide + doxorubicin + cisplatin, $n=7$; paclitaxel + carboplatin, $n=7$; ifosfamide + cisplatin + etoposide, $n=4$; and etoposide + cisplatin, $n=4$. Patients' characteristics are listed in Table 1.

\section{Response data and survival analysis}

The median number of pemetrexed chemotherapy cycles was four (range, 1-6). Of the 22 patients, partial response was confirmed in five and ten showed stable disease, representing a response rate of $22.7 \%$ and disease control rate of $68.2 \%$. The median PFS was 4.5 months (95\% confidence interval [CI]: 3.1-5.9) (Figure 1). There was no significant association between PFS and sex $(P=0.18)$, age $(P=0.29)$, stage $(P=0.74)$, and chemotherapy regimen $(P=0.69)$. However, a trend of longer PFS existed in thymoma $(P=0.083)$ and PS of $0-1$ $(P=0.053)$ (Table 2).

The median OS was 34.9 months (95\% CI: 31.3-38.4) (Figure 2). No OS difference was according to sex $(P=0.18)$, age $(P=0.29)$, stage $(P=0.74)$, and chemotherapy regimen $(P=0.85)$. The OS was longer in patients with thymoma than thymic carcinoma $(P=0.039)$ (Table 2$)$.

\section{Thymidylate synthetase mRNA level}

The median TS mRNA levels in all patients were $380.5 \pm$ $326.0 \times 10^{-4}$. TS mRNA levels in patients with disease control (partial response + stable disease) under pemetrexed treatment were lower than those with progressive 
Table I Clinical characteristics of 22 patients with advanced thymic epithelial tumors

\begin{tabular}{|c|c|c|c|c|c|c|c|c|c|}
\hline Case & $\begin{array}{l}\text { Sex/age, } \\
\text { years }\end{array}$ & Histology & Stage & PS & $\begin{array}{l}\text { Line } \\
\text { of therapy }\end{array}$ & Regimen & Response & $\begin{array}{l}\text { PFS, } \\
\text { months }\end{array}$ & $\begin{array}{l}\text { OS, } \\
\text { months }\end{array}$ \\
\hline I & $F / 69$ & Thymic carcinoma & Iva & 1 & Second & PC & SD & 4.0 & 29.5 \\
\hline 2 & $M / 48$ & Thymic carcinoma & $\mathrm{IVb}$ & 2 & Third & Pemetrexed & SD & 4.0 & 28.7 \\
\hline 3 & $M / 55$ & Thymoma & $\mathrm{IVb}$ & 1 & Second & Pemetrexed & PR & 7.0 & 32.5 \\
\hline 4 & $\mathrm{~F} / 49$ & Thymoma & Iva & 2 & Second & PC & PR & 9.5 & 37.4 \\
\hline 5 & $\mathrm{~F} / 47$ & Thymic carcinoma & $\mathrm{IVb}$ & 2 & Third & Pemetrexed & PD & 1.5 & 19.0 \\
\hline 6 & $M / 55$ & Thymoma & $\mathrm{IVb}$ & 1 & Second & Pemetrexed & $P R$ & 10.5 & 39.0 \\
\hline 7 & $\mathrm{~F} / 60$ & Thymic carcinoma & $\mathrm{IVb}$ & 2 & Third & PC & PD & 1.2 & 17.0 \\
\hline 8 & $F / 22$ & Thymic carcinoma & Iva & 1 & Third & Pemetrexed & SD & 8.0 & 35.5 \\
\hline 9 & $\mathrm{~F} / 70$ & Thymoma & $\mathrm{IVb}$ & 2 & Fourth & Pemetrexed & SD & 5.5 & $44.0+$ \\
\hline 10 & $M / 65$ & Thymoma & $\mathrm{IVb}$ & 1 & Second & Pemetrexed & SD & 6.0 & 35.0 \\
\hline II & $\mathrm{F} / 45$ & Thymic carcinoma & Iva & 2 & Fifth & Pemetrexed & PD & 1.4 & 20.0 \\
\hline 12 & $M / 27$ & Thymic carcinoma & $\mathrm{IVb}$ & 2 & Third & Pemetrexed & PD & 1.0 & 15.0 \\
\hline 13 & $M / 32$ & Thymoma & $\mathrm{IVb}$ & I & Fifth & Pemetrexed & SD & 3.5 & 25.6 \\
\hline 14 & $M / 39$ & Thymic carcinoma & Iva & 2 & Third & Pemetrexed & PD & 2.2 & 23.5 \\
\hline 15 & $\mathrm{~F} / 55$ & Thymic carcinoma & Iva & 0 & Fifth & PC & SD & 6.0 & 45.8 \\
\hline 16 & $M / 59$ & Thymic carcinoma & $\mathrm{IVb}$ & I & Third & Pemetrexed & SD & 6.0 & 36.6 \\
\hline 17 & $\mathrm{~F} / 45$ & Thymoma & $\mathrm{IVb}$ & 0 & Fourth & Pemetrexed & PD & 1.2 & 47.4 \\
\hline 18 & $\mathrm{~F} / 47$ & Thymic carcinoma & Iva & 2 & Second & Pemetrexed & PD & 1.0 & $12.3+$ \\
\hline 19 & $M / 56$ & Thymic carcinoma & $\mathrm{IVb}$ & 0 & Fifth & Pemetrexed & SD & 5.0 & 37.2 \\
\hline 20 & $\mathrm{~F} / 43$ & Thymoma & $\mathrm{IVb}$ & 2 & Fourth & Pemetrexed & PR & 4.5 & $23.6+$ \\
\hline 21 & $M / 44$ & Thymic carcinoma & Iva & 0 & Second & Pemetrexed & PR & 6.5 & 32.8 \\
\hline 22 & $\mathrm{~F} / 49$ & Thymic carcinoma & $\mathrm{IVb}$ & 1 & Fifth & Pemetrexed & SD & 4.5 & 34.9 \\
\hline
\end{tabular}

Abbreviations: PS, performance status; M, male; F, female; PC, pemetrexed/carboplatin; PFS, progression-free survival; OS, overall survival; PR, partial response; SD, stable disease; PD, progressive disease.

disease $\left(268.0 \pm 160.5 \times 10^{-4}\right.$ vs $567.0 \pm 445.0 \times 10^{-4}$ $P=0.065$ ) (Figure 3).

\section{Discussion}

Our results demonstrated that some patients with advanced TET may benefit from pemetrexed-based regimen and the clinical efficacy of pemetrexed in TET was associated with TS mRNA level. To our knowledge, we represent the largest

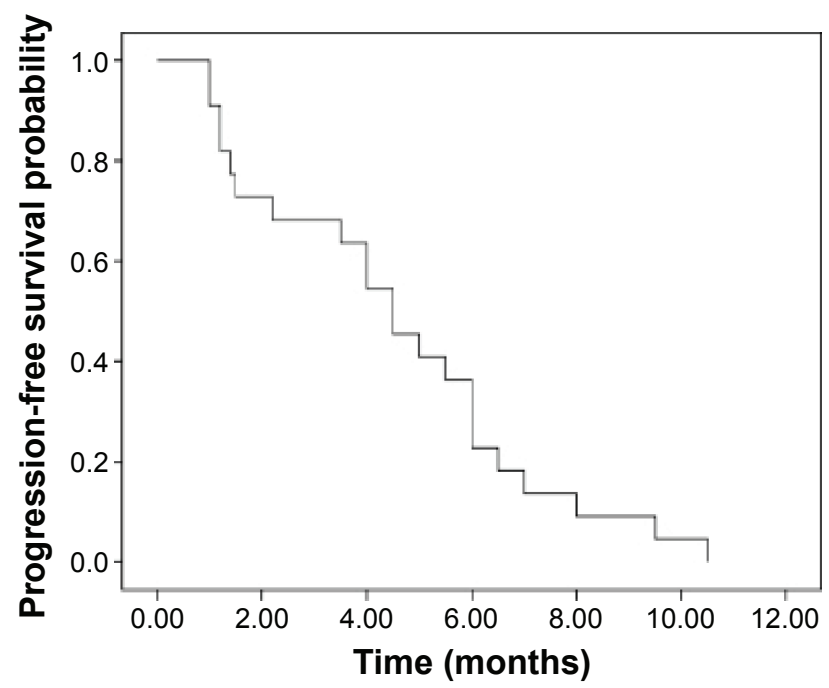

Figure I Progression-free survival curve of 22 patients with pemetrexed-based chemotherapy. amount of data to assess the efficacy of pemetrexed-based regimen as salvage chemotherapy in TET.

Anthracycline- and platinum-based regimens are now recommended as first-line treatment in the National Comprehensive Cancer Network (NCCN) guidelines. ${ }^{16}$ For patients with TET who have progressed after chemotherapy, no standard treatment is established currently. All of the previous regimens were based on case reports or series with limited numbers. The regimens included S-1, gemcitabine, irinotecan, and docetaxel, ${ }^{17-20}$ and the efficacy of these regimens varied from 3 to 18 months.

Pemetrexed-based chemotherapy is widely used in NSCLC patients and demonstrated better efficacy and lower toxicity than other third-generation drugs. ${ }^{9-11}$ However, little is known about the efficacy of pemetrexed in TET due to its rarity. Liang et al reported 16 patients with TET (six with thymoma and ten with thymic carcinoma) and demonstrated that the median PFS was different in thymoma (13.8 months) and thymic carcinoma (6.5 months) with pemetrexed-based treatment. ${ }^{21}$ Our results showed that the median PFS was 4.5 months with a regimen of pemetrexed-based treatment. The reason for shorter PFS in this study may be due to more patients with poor PS and further-line treatment in the current cohort.

Previous studies demonstrated that negative or low level TS expression benefited more from pemetrexed-based 
Table 2 Univariate analysis of patients with pemetrexed-based chemotherapy

\begin{tabular}{lcccc}
\hline & PFS & P-value & Overall survival & P-value \\
\hline Sex & & 0.39 & & 0.18 \\
$\quad$ Male & 5.0 & & 32.5 & \\
$\quad$ Female & 4.0 & & 35.5 & \\
Age, years & & 0.17 & & 0.29 \\
$\quad<50$ & 3.5 & & 28.7 & \\
$\quad \geq 50$ & 6.0 & & 36.6 & \\
Histology & & 0.083 & & 0.039 \\
$\quad$ Thymoma & 5.5 & & 37.4 & \\
$\quad$ Thymic carcinoma & 4.0 & & 29.5 & 0.20 \\
Performance status & & 0.053 & & \\
$\quad$ 0-I & 6.0 & & 35.0 & \\
$\quad 2$ & 1.5 & & 23.5 & \\
Stage & & 0.68 & & \\
$\quad$ Iva & 4.0 & & 34.9 & \\
$\quad$ Ivb & 4.5 & & 32.8 & \\
Chemotherapy regimen & & 0.69 & & \\
$\quad$ Pemetrexed & 4.5 & & 34.9 & \\
$\quad$ Pemetrexed/carboplatin & 4.0 & & 29.5 & \\
\hline
\end{tabular}

Abbreviation: PFS, progression-free survival.

chemotherapy than positive or high levels of TS in NSCLC patients. ${ }^{12,13}$ Although some studies reported the expression of TS level in thymic malignancies, no association between TS level and pemetrexed's efficacy has been investigated. ${ }^{22,23}$ We examined the TS mRNA level in all of our 22 patients. The results demonstrated that patients with lower TS mRNA level benefited more from pemetrexed-based regimen. Our data indicated that TS mRNA level enables the selection of pemetrexed-based regimen options in TET patients.

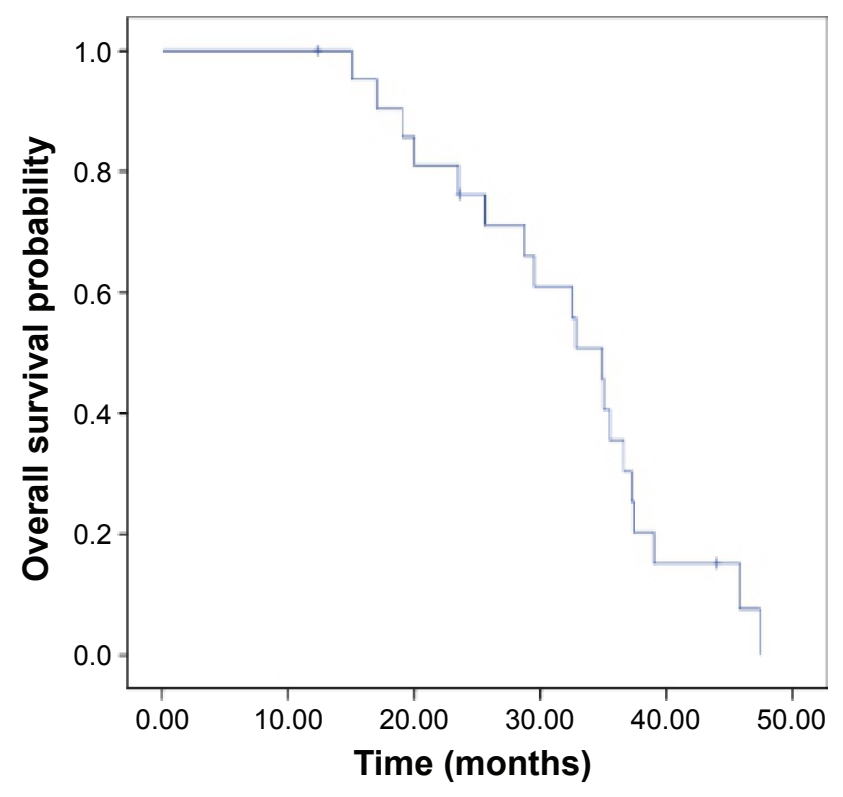

Figure 2 Overall survival curve of 22 patients with pemetrexed-based chemotherapy.

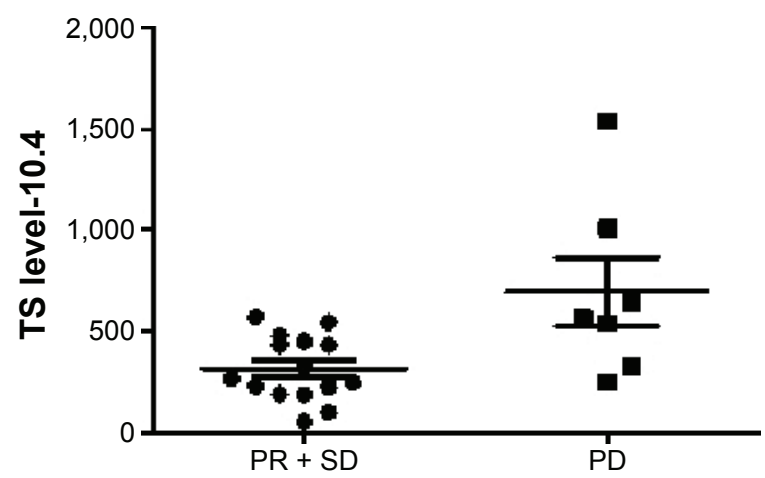

Figure 3 Efficacy of pemetrexed treatment in patients with different levels of thymidylate synthetase mRNA.

Abbreviations: TS, thymidylate synthetase; PR, partial response; SD, stable disease; PD, progressive disease.

Some of the limitations of our study were related to its retrospective design with an enrollment of only 22 patients. However, given the limited literature available, our study can also be meaningful for clinical practice.

In summary, our results suggest that pemetrexed-based treatment is effective as a salvage chemotherapy for pretreated advanced TET. The TS mRNA level may be a predicting factor for the efficacy of pemetrexed-based regimen in TET.

\section{Disclosure}

The authors report no conflicts of interest to in this work.

\section{References}

1. Engels EA, Pfeiffer RM. Malignant thymoma in the United States: demographic patterns in incidence and associations with subsequent malignancies. Int J Cancer. 2003;105(4):546-551.

2. Song Z, Zhang Y. Outcomes after surgical resection of thymic carcinoma: A study from a single tertiary referral centre. Eur J Surg Oncol. 2014; 40(11):1523-1527.

3. Hishida T, Nomura S, Yano M, et al. Long-term outcome and prognostic factors of surgically treated thymic carcinoma: results of 306 cases from a Japanese Nationwide Database Study. Eur J Cardiothorac Surg. 2015; 49(3):835-841.

4. Wei ML, Kang D, Gu L, Qiu M, Zhengyin L, Mu Y. Chemotherapy for thymic carcinoma and advanced thymoma in adults. Cochrane Database Syst Rev. 2013;8:CD008588.

5. Schmitt J, Loehrer PJ Sr. The role of chemotherapy in advanced thymoma. J Thorac Oncol. 2010;5(10 Suppl 4):S357-S360.

6. Loehrer PJ Sr, Kim K, Aisner SC, et al. Cisplatin plus doxorubicin plus cyclophosphamide in metastatic or recurrent thymoma: final results of an intergroup trial. The Eastern Cooperative Oncology Group, Southwest Oncology Group, and Southeastern Cancer Study Group. J Clin Oncol. 1994;12(6):1164-1168.

7. Fornasiero A, Daniele O, Ghiotto C, et al. Chemotherapy of invasive thymoma. J Clin Oncol. 1990;8(8):1419-1423.

8. Loehrer PJ Sr, Jiroutek M, Aisner S, et al. Combined etoposide, ifosfamide, and cisplatin in the treatment of patients with advanced thymoma and thymic carcinoma: an intergroup trial. Cancer. 2001;91(11): 2010-2015.

9. Hanna N, Shepherd FA, Fossella FV, et al. Randomized phase III trial of pemetrexed versus docetaxel in patients with non-small-cell lung cancer previously treated with chemotherapy. J Clin Oncol. 2004;22(9): $1589-1597$. 
10. Scagliotti GV, Parikh P, von Pawel J, et al. Phase III study comparing cisplatin plus gemcitabine with cisplatin plus pemetrexed in chemotherapy-naive patients with advanced-stage non-small-cell lung cancer. J Clin Oncol. 2008;26(21):3543-3551.

11. Paz-Ares L, de Marinis F, Dediu M, et al. Maintenance therapy with pemetrexed plus best supportive care versus placebo plus best supportive care after induction therapy with pemetrexed plus cisplatin for advanced non-squamous non-small-cell lung cancer (PARAMOUNT): a double-blind, phase 3, randomised controlled trial. Lancet Oncol. 2012;13(3):247-255.

12. Sun JM, Ahn JS, Jung SH, et al. Pemetrexed plus cisplatin versus gemcitabine plus cisplatin according to thymidylate synthase expression in nonsquamous non-small-cell lung cancer: a biomarkerstratified randomized phase II trial. J Clin Oncol. 2015;33(22): 2450-2456.

13. Nicolson MC, Fennell DA, Ferry D, et al. Thymidylate synthase expression and outcome of patients receiving pemetrexed for advanced nonsquamous non-small-cell lung cancer in a prospective blinded assessment phase II clinical trial. J Thorac Oncol. 2013;8(7):930-939.

14. Ren S, Chen X, Kuang P, et al. Association of EGFR mutation or ALK rearrangement with expression of DNA repair and synthesis genes in never-smoker women with pulmonary adenocarcinoma. Cancer. 2012;118(22):5588-5594.

15. Bepler G, Sommers KE, Cantor A, et al. Clinical efficacy and predictive molecular markers of neoadjuvant gemcitabine and pemetrexed in resectable non-small cell lung cancer. J Thorac Oncol. 2008;3(10): 1112-1118.
16. Ettinger DS, Riely GJ, Akerley W, et al. NCCN clinical practice guidelines in oncology: thymomas and thymic carcinomas. Version 1; 2014 Available from: https://www.nccn.org/professionals/physician_gls/pdf/ thymic.pdf. Accessed June 2, 2016.

17. Palmieri G, Merola G, Federico P, et al. Preliminary results of phase II study of capecitabine and gemcitabine (CAP-GEM) in patients with metastatic pretreated thymic epithelial tumors (TETs). Ann Oncol. 2010; 21(6):1168-1172.

18. Okuma Y, Shimokawa T, Takagi Y, et al. S-1 is an active anticancer agent for advanced thymic carcinoma. Lung Cancer. 2010;70(3):357-363.

19. Song Z. Chemotherapy with paclitaxel plus carboplatin for relapsed advanced thymic carcinoma. J Thorac Dis. 2014;6(12):1808-1812.

20. Song Z, Yu X, He C, Zhang B, Zhang Y. Docetaxel-based chemotherapy as second-line regimen for advanced thymic carcinoma. Thorac Cancer. 2014;5(2):169-173.

21. Liang Y, Padda SK, Riess JW, West RB, Neal JW, Wakelee HA. Pemetrexed in patients with thymic malignancies previously treated with chemotherapy. Lung Cancer. 2015;87(1):34-38.

22. Kaira K, Serizawa M, Koh Y, et al. Expression of thymidylate synthase, orotate phosphoribosyltransferase and dihydropyrimidine dehydrogenase in thymic epithelial tumors. Lung Cancer. 2011;74(3):419-425.

23. Yokota K, Sasaki H, Okuda K, et al. Expression of thymidylate synthase and orotate phosphoribosyltransferase in thymic carcinoma. Exp Ther Med. 2012;4(4):589-593.
OncoTargets and Therapy

\section{Publish your work in this journal}

OncoTargets and Therapy is an international, peer-reviewed, open access journal focusing on the pathological basis of all cancers, potential targets for therapy and treatment protocols employed to improve the management of cancer patients. The journal also focuses on the impact of management programs and new therapeutic agents and protocols on

\section{Dovepress}

patient perspectives such as quality of life, adherence and satisfaction. The manuscript management system is completely online and includes a very quick and fair peer-review system, which is all easy to use. Visit http://www.dovepress.com/testimonials.php to read real quotes from published authors. 